\title{
Riemann-Liouville fractional stochastic evolution equations driven by both Wiener process and fractional Brownian motion
}

\section{Min Yang ${ }^{1}$ and Haibo $\mathrm{Gu}^{2^{*}}$}

"Correspondence:

hbgu_math@163.com

${ }^{2}$ School of Mathematics Sciences,

Xinjiang Normal University, 830017

Urumai, China

Full list of author information is

available at the end of the article

\section{Springer}

\begin{abstract}
This article is devoted to the study of the existence and uniqueness of mild solution to a class of Riemann-Liouville fractional stochastic evolution equations driven by both Wiener process and fractional Brownian motion. Our results are obtained by using fractional calculus, stochastic analysis, and the fixed-point technique. Moreover, an example is provided to illustrate the application of the obtained abstract results.
\end{abstract}

Keywords: Riemann-Liouville fractional derivative; Stochastic evolution equations; Fractional Brownian motion; Mild solution

\section{Introduction}

Fractional calculus has gained considerable popularity during the past decades since it has been recognized as one of the best tools to model physical systems possessing longterm memory and long-range spatial interactions, which also plays an important role in diverse areas of science and engineering, as well as other applied sciences. As a result of the intensive development of fractional calculus, there has been a significant breakthrough in theoretical analysis and applications of fractional differential equations in the literature $[8,14,15,17,23]$. In recent decades, the existence and uniqueness of mild solutions, as well as controllability for fractional differential equations with Caputo fractional derivative, have attracted much attention. We can refer to $[1-3,11,13,16,18-20,24,25]$ and the references therein.

It is worth mentioning that by applying Laplace transform and probability density functions, Zhou et al. [26] gave a suitable concept of mild solutions for a class of RiemannLiouville fractional evolution equations with nonlocal conditions. Li et al. [10] considered the Cauchy problems for fractional differential equations with Riemann-Liouville fractional derivatives by using the $\alpha$-resolvent operator. Yang and Wang [22] investigated the approximate controllability of Riemann-Liouville fractional differential inclusions. For more details about the existence of mild solutions of Riemann-Liouville fractional differential equations, one can refer to $[12,20]$. On the other hand, Ahmed and El-Borai [1] studied Hilfer fractional stochastic integro-differential equations. We can refer to [5-7]

c) The Author(s) 2021. This article is licensed under a Creative Commons Attribution 4.0 International License, which permits use, sharing, adaptation, distribution and reproduction in any medium or format, as long as you give appropriate credit to the original author(s) and the source, provide a link to the Creative Commons licence, and indicate if changes were made. The images or other third party material in this article are included in the article's Creative Commons licence, unless indicated otherwise in a credit line to the material. If material is not included in the article's Creative Commons licence and your intended use is not permitted by statutory regulation or exceeds the permitted use, you will need to obtain permission directly from the copyright holder. To view a copy of this licence, visit http://creativecommons.org/licenses/by/4.0/. 
for more details about the existence of mild solutions and approximate controllability of Hilfer fractional differential equations and inclusions.

As an generalization of Brownian motion, fractional Brownian motion has received a lot of attention in the recent years, which as we know is a Gaussian process with selfsimilarity and stationary increments, as well as long-range dependence properties. When Hurst parameter $H \in\left(0, \frac{1}{2}\right)$, fractional Brownian motion is neither a semimartingale nor a Markov process. Hence, the classical stochastic analysis techniques are invalid to study it. Recently, the research of stochastic differential equations driven by fractional Brownian motion has been investigated by many authors, see $[4,9,21,28]$ and the references therein.

However, we would like to emphasize that it is natural and also important to study the existence of mild solutions for Riemann-Liouville fractional stochastic evolution equations driven by both Wiener process and fractional Brownian motion since it has not yet been sufficiently studied in contrast with the integer-order case. In this paper we are concerned with the following Riemann-Liouville fractional stochastic evolution equations with nonlocal conditions driven by both Wiener process and fractional Brownian motion:

$$
\left\{\begin{aligned}
{ }^{L} \mathcal{D}^{\alpha}[x(t)-h(t, x(t))]= & A x(t)+F(t, x(t)) \frac{d \omega(t)}{d t} \\
& +\sigma(t) \frac{d B_{Q}^{H}(t)}{d t}, \quad t \in J^{\prime}=(0, b], \\
\mathcal{I}_{0^{+}}^{1-\alpha}[x(0)-g(x)]=x_{0} & \in X,
\end{aligned}\right.
$$

where ${ }^{L} \mathcal{D}_{t}^{\alpha}$ denotes the Riemann-Liouville fractional derivative in time defined for $\frac{1}{2}<$ $\alpha<1, \mathcal{I}^{\alpha}$ is the temporal Riemann-Liouville fractional integral operator of order $\alpha ; x(\cdot)$ takes values in a separable Hilbert space $X, A$ is the infinitesimal generator of an analytic semigroup $\{S(t)\}_{t \geq 0}$ on a real separable Hilbert space $X$ with inner product $\langle\cdot, \cdot\rangle$ and norm $\|\cdot\|$. Assume $K$ to be another separable Hilbert space with inner product $\langle\cdot, \cdot\rangle$ and norm $\|\cdot\|_{K}$. Let $L(K, X)$ denote the space of all bounded linear operators from $K$ to $X$, $h: J \times X \rightarrow X$ and $F: J \times X \rightarrow L(K, X)$ be functions satisfying some specific assumptions given in $\left(\mathrm{H}_{2}\right)-\left(\mathrm{H}_{3}\right) ;\{\omega(t)\}_{t \geq 0}$ is a given $K$-valued Wiener process with a finite trace nuclear covariance operator $Q>0$ defined on the filtered complete probability space $(\Omega, \mathcal{F}, P) ; B^{H}$ is a fractional Brownian motion with Hurst parameter $H \in\left(\frac{1}{2}, 1\right)$. The initial data $x_{0}$ is an $\mathcal{F}_{0}$-measurable, stochastic process independent of the Wiener process $\omega$ and $\mathrm{fBm} B^{H}(t)$ with finite second moment.

A brief outline of this paper is given as follows. In Sect. 2, we recall some notations and preliminaries about fractional Brownian motion and fractional calculus. Using the fixed point theorem, Sect. 3 establishes the existence of mild solutions for system (1.1). In Sect. 4, we will give an example to illustrate the application of the obtained abstract results. Conclusions and discussions are given in the final section.

\section{Preliminaries}

In this section, we first present some notations, definitions, and preliminary facts.

We first recall some basic facts about $\mathrm{fBm}$ and the Wiener integral with respect to $\mathrm{fBm}$. Let $(\Omega, \mathcal{F}, P)$ be a complete probability space. We choose a time interval $J=[0, b]$ with arbitrary fixed horizon $b$, assume $\left\{B^{H}(t), t \in J\right\}$ to be a one-dimensional fractional Brownian motion with Hurst parameter $H \in(0,1)$. The definition implies that $B^{H}$ is a continuous 
and centered Gaussian process with covariance function

$$
R_{H}(s, t)=\frac{1}{2}\left(t^{2 H}+s^{2 H}-|t-s|^{2 H}\right) .
$$

In what follows, we always assume $\frac{1}{2}<H<1$. Consider the square integrable kernel

$$
K_{H}(s, t)=c_{H} s^{\frac{1}{2}-H} \int_{s}^{t}(u-s) u^{H-\frac{1}{2}} d u
$$

where $c_{H}=\left[\frac{H(2 H-1)}{\beta\left(2-2 H, H-\frac{1}{2}\right)}\right]^{\frac{1}{2}}, t>s$. Then we have

$$
\frac{\partial K_{H}}{\partial t}(t, s)=c_{H}\left(\frac{t}{s}\right)^{H-\frac{1}{2}}(t-s)^{H-\frac{3}{2}}
$$

We will denote by $\mathcal{H}$ the reproducing kernel Hilbert space of the $\mathrm{fBm}$. In fact, $\mathcal{H}$ is the closure of the linear space of indicator functions $\left\{I_{[0, t]}, t \in[0, T]\right\}$ with respect to the scalar product

$$
\left\langle I_{[0, t]}, I_{[0, s]}\right\rangle_{\mathcal{H}}=R_{H}(t, s) .
$$

The mapping $I_{[0, t]} \rightarrow B^{H}(t)$ can be extended to an isometry between $\mathcal{H}$ and the first Wiener chaos, and we denote by $B^{H}(\varphi)$ the image of $\varphi$ under this isometry.

Set $K_{H}^{*}$ be the linear operator from $\mathcal{H}$ to $L^{2}([0, b])$ defined by

$$
\left(K_{H}^{*} \varphi\right)(s)=\int_{s}^{t} \varphi(t) \frac{\partial K_{H}}{\partial t}(t, s) d t
$$

Recall that the operator $K_{H}^{*}$ is an isometry between $\mathcal{H}$ and $L^{2}([0, b])$.

Let the process $\omega=\omega(t), t \in[0, b]$ be defined by

$$
\omega(t)=B^{H}\left(\left(K_{H}^{*}\right)^{-1} I_{[0, b])}\right)
$$

Then $\omega$ is a Wiener process, while $B^{H}$ has the integral representation

$$
B^{H}(t)=\int_{0}^{t} K_{H}(t, s) d \omega(s)
$$

We recall that for $\varphi, \psi$ on $[0, b]$, their scalar product in $\mathcal{H}$ is given by

$$
\langle\varphi, \psi\rangle_{\mathcal{H}}=\alpha_{H} \int_{0}^{b} \int_{0}^{b} \varphi(r) \psi(u)|r-u|^{2 H-2} d u d r<\infty
$$

where $\alpha_{H}=H(2 H-1)$.

The embedding relationship is as follows [21]:

$$
L^{1}([0, b]) \subset L^{\frac{1}{\mathcal{H}}} \subset|\mathcal{H}| \subset \mathcal{H} .
$$


Assume that $Y$ is a real separable Hilbert space. Suppose that there exists a complete orthogonal system $\left\{e_{n}\right\}_{n=1}^{\infty}$ in $Y$. Let $Q \in L(Y, Y)$ be an operator with finite trace $\operatorname{Tr} Q=\sum_{n=1}^{\infty} \lambda_{n}<\infty\left(\lambda_{n} \geq 0\right)$ such that $Q e_{n}=\lambda_{n} e_{n}$. By using the covariance operator $Q$, the infinite-dimensional fBM on $Y$ can be defined as

$$
B^{H}(t)=B_{Q}^{H}(t)=\sum_{n=1}^{\infty} \sqrt{\lambda_{n}} \varphi e_{n} B_{n}^{H}(t)
$$

where $B_{n}^{H}(t)$ are one-dimensional standard fractional Brownian motions mutually independent on $(\Omega, \mathcal{F}, P)$. Assume that the space $\mathcal{L}_{2}^{0}:=\mathcal{L}_{2}^{0}(Y, X)$ consists of all $Q$-HilbertSchmidt operators $\varphi: Y \rightarrow X$. Recall that $\varphi \in L(Y, X)$ is called a Q-Hilbert-Schmidt operator if

$$
\|\varphi\|_{\mathcal{L}_{2}^{0}}^{2}:=\sum_{n=1}^{\infty}\left\|\sqrt{\lambda_{n}} \varphi e_{n}\right\|^{2}<\infty
$$

and that the space $\mathcal{L}_{2}^{0}$ equipped with the inner product $\langle\varphi, \psi\rangle_{\mathcal{L}_{2}^{0}}=\sum_{n=1}^{\infty}\left\langle\varphi e_{n}, \psi e_{n}\right\rangle$ is a separable Hilbert space.

Let $(\phi(s))_{s \in[0, b]}$ be a deterministic function with values in $\mathcal{L}_{2}^{0}(Y, X)$. The stochastic integral of $\phi$ with respect to $B^{H}$ is defined as

$$
\int_{0}^{t} \phi(s) d B^{H}(s)=\sum_{n=1}^{\infty} \int_{0}^{t} \sqrt{\lambda_{n}}\left(K_{H}^{*}\left(\phi e_{n}\right)\right)(s) d B_{n}(s)
$$

Lemma 2.1 ([4]) If $\varphi:[0, b] \rightarrow \mathcal{L}_{2}^{0}(Y, X)$ satisfies $\int_{0}^{b}\|\varphi(s)\|_{\mathcal{L}_{2}^{0}}^{2} d s<\infty$, then the aforementioned sum in (2.1) is well defined as an $X$-valued random variable, and we have

$$
E\left\|\int_{0}^{t} \varphi(s) d B^{H}(s)\right\|^{2} \leq c_{0} H(2 H-1) t^{2 H-1} \int_{0}^{t}\|\varphi(s)\|_{\mathcal{L}_{2}^{0}}^{2} d s .
$$

We next denote by $\mathcal{L}_{2}(\Omega, X)$ the collection of all strongly-measurable, square-integrable, $X$-valued random variables. Obviously, $\mathcal{L}_{2}(\Omega, X)$ is a Banach space equipped with norm $\|x(\cdot)\|_{\mathcal{L}_{2}(\Omega, X)}=(E|x(\cdot)|)^{\frac{1}{2}}$. Let $C\left(J, \mathcal{L}_{2}(\Omega, X)\right)$ denote the Banach space of all $X$-valued continuous functions from $J=[0, b]$ into $\mathcal{L}_{2}(\Omega, X)$ satisfying $\sup _{t \in J}\|x(t)\|^{2}<\infty$. Let $J^{\prime}=(0, b]$. To define the mild solution of system (1.1), we also need to consider the Banach space $C_{\alpha}(J, X)=\left\{x: t^{1-\alpha} x(t) \in C\left(J, \mathcal{L}_{2}(\Omega, X)\right)\right\}$ with the norm

$$
\|x\|_{C_{\alpha}}=\left(\sup _{t \in J} E\left\|t^{1-\alpha} x(t)\right\|^{2}\right)^{\frac{1}{2}}
$$

Throughout this paper, we assume that $0 \in \rho(A)$ where $\rho(A)$ denotes the resolvent set of $A$. Then it is possible for us to define the fractional power $A^{\eta}$ as a closed linear operator on its domain $D\left(A^{\eta}\right)$ for $0<\eta \leq 1$ (see [25]). For an analytic semigroup $\{S(t)\}_{t \geq 0}$, the following properties hold:

(i) There exists $M \geq 1$ such that

$$
M:=\sup _{t \in[0,+\infty)} S(t)<\infty \text {; }
$$


(ii) For $\forall \eta \in(0,1]$, there exists a positive constant $C_{\eta}$ such that

$$
\left\|A^{\eta} S(t)\right\| \leq \frac{C_{\eta}}{t^{\eta}}, \quad 0<t \leq b .
$$

For further convenience, set

$$
\mathcal{S}_{\alpha}(t) x=\int_{0}^{\infty} \phi_{\alpha}(\theta) S\left(t^{\alpha} \theta\right) x d \theta, \quad \mathcal{P}_{\alpha}(t) x=\int_{0}^{\infty} \alpha \theta \phi_{\alpha}(\theta) S\left(t^{\alpha} \theta\right) x d \theta
$$

where

$$
\phi_{\alpha}(\theta)=\frac{1}{\alpha} \theta^{-1-\frac{1}{\alpha}} \psi_{\alpha}\left(\theta^{\frac{-1}{\alpha}}\right),
$$

which involves the Wright-type function

$$
\psi_{\alpha}(\theta)=\frac{1}{\pi} \sum_{n=1}^{\infty}(-1)^{n-} \theta^{-\alpha n-1} \frac{\Gamma(n \alpha+1)}{n !} \sin (n \pi \alpha), \quad \theta \in(0, \infty)
$$

connected with the following one-sided stable probability function: $\phi_{\alpha}(\theta) \geq 0, \theta \in(0, \infty)$ and $\int_{0}^{\infty} \phi_{\alpha}(\theta) d \theta=1$.

Lemma 2.2 ([23]) The operators $\mathcal{S}_{\alpha}$ and $\mathcal{P}_{\alpha}$ have the following properties:

(i) For any fixed $t \geq 0, \mathcal{S}_{\alpha}(t)$ and $\mathcal{P}_{\alpha}(t)$ are linear and bounded operators, i.e., for any $x \in X$,

$$
\left\|\mathcal{S}_{\alpha}(t) x\right\| \leq M\|x\| \quad \text { and } \quad\left\|\mathcal{P}_{\alpha}(t) x\right\| \leq \frac{M}{\Gamma(\alpha)}\|x\| .
$$

(ii) $\left\{\mathcal{S}_{\alpha}(t)\right\}_{t \geq 0}$ and $\left\{\mathcal{P}_{\alpha}(t)\right\}_{t \geq 0}$ are strongly continuous.

(iii) For every $t>0, \mathcal{S}_{\alpha}(t)$ and $\mathcal{P}_{\alpha}(t)$ are also compact operators if $S(t), t>0$ is compact.

Lemma $2.3([25,27])$ For any $t>0$ and $0 \leq \gamma<1$, there exists a positive constant $C_{\gamma}$ such that

$$
A \mathcal{P}_{\alpha}(t) x=A^{1-\gamma} \mathcal{P}_{\alpha}(t) A^{\gamma} x
$$

and

$$
\left\|A^{\gamma} \mathcal{P}_{\alpha}(t)\right\| \leq \frac{\alpha C_{\gamma} \Gamma(2-\gamma)}{t^{\alpha \gamma} \Gamma(1+\alpha(1-\gamma))}
$$

Lemma 2.4 ([23]) For $\sigma \in(0,1]$ and $0<a \leq b$, we have $\left|a^{\sigma}-b^{\sigma}\right| \leq(b-a)^{\sigma}$.

\section{Lemma 2.5 If}

$$
\begin{aligned}
x(t)= & \frac{t^{\alpha-1}}{\Gamma(\alpha)}\left[x_{0}+h(0, x(0))+g(x)\right]+h(t, x(t))+\frac{1}{\Gamma(\alpha)} \int_{0}^{t}(t-s)^{\alpha-1} A x(s) d s \\
& +\frac{1}{\Gamma(\alpha)} \int_{0}^{t}(t-s)^{\alpha-1} F(s, x(s)) d \omega(s)+\frac{1}{\Gamma(\alpha)} \int_{0}^{t}(t-s)^{\alpha-1} \sigma(s) d B_{Q}^{H}(s), \quad t>0,
\end{aligned}
$$


then we have

$$
\begin{aligned}
x(t)= & t^{\alpha-1} \mathcal{P}_{\alpha}(t)\left[x_{0}+h(0, x(0))+g(x)\right]+h(t, x(t)) \\
& +\int_{0}^{t}(t-s)^{\alpha-1} \mathcal{P}_{\alpha}(t-s) F(s, x(s)) d \omega(s) \\
& +\int_{0}^{t}(t-s)^{\alpha-1} A \mathcal{P}_{\alpha}(t-s) h(s, x(s)) d s \\
& +\int_{0}^{t}(t-s)^{\alpha-1} \mathcal{P}_{\alpha}(t-s) \sigma(s) d B_{Q}^{H}(s), \quad t>0 .
\end{aligned}
$$

Proof Referring to [25], we omit the proof here.

\section{Main results}

In this section, we present and prove the existence of mild solutions for system (1.1). To develop our results, we first give the concept of mild solution for system (1.1).

Definition 3.1 An $\mathcal{F}_{t}$-adapted and measurable stochastic process $x \in C_{\alpha}(J, X)$ is said to be a mild solution of system (1.1) if $x_{0}, g \in \mathcal{L}_{2}^{0}(\Omega, X)$, for each $s \in[0, b)$, the function $(t-$ $s)^{\alpha-1} A T_{\alpha}(t-s) h(s, x(s))$ is integrable and the following integral equation is verified:

$$
\begin{aligned}
x(t)= & t^{\alpha-1} \mathcal{P}_{\alpha}(t)\left[x_{0}+h(0, x(0))+g(x)\right]+h(t, x(t)) \\
& +\int_{0}^{t}(t-s)^{\alpha-1} \mathcal{P}_{\alpha}(t-s) F(s, x(s)) d \omega(s) \\
& +\int_{0}^{t}(t-s)^{\alpha-1} A \mathcal{P}_{\alpha}(t-s) h(s, x(s)) d s \\
& +\int_{0}^{t}(t-s)^{\alpha-1} \mathcal{P}_{\alpha}(t-s) \sigma(s) d B_{Q}^{H}(s), \quad t \in J^{\prime} .
\end{aligned}
$$

For our readers' convenience, we first introduce some notations:

$$
\begin{aligned}
& \left\|A^{-\beta}\right\|=M_{0}, \quad K(\alpha, \beta)=\frac{C_{1-\beta} \Gamma(1+\beta)}{\beta \Gamma(1+\alpha \beta)}, \quad c=\frac{\alpha-1}{1-\alpha_{1}}, \quad \Lambda=\frac{b^{(1+c)\left(2-2 \alpha_{1}\right)}}{(1+c)^{2-2 \alpha_{1}}}, \\
& \alpha_{1} \in\left[\frac{1}{2}, \alpha\right) .
\end{aligned}
$$

To establish the main results, we require the following hypotheses:

$\left(\mathrm{H}_{1}\right)$ Semigroup $S(t)$ is compact for each $t>0$;

$\left(\mathrm{H}_{2}\right) \quad$ (2a) for each $x \in X$, the function $F(\cdot, x): J \rightarrow \mathcal{L}_{2}^{0}(X, Y)$ is strongly measurable with respect to $t$, and for each $t \in J$, the function $F(t, \cdot): X \rightarrow \mathcal{L}_{2}^{0}(X, Y)$ is continuous with respect to $x$;

(2b) there exist a function $N(t) \in L^{\frac{1}{2 \alpha_{1}-1}}(J), \alpha_{1} \in\left[\frac{1}{2}, \alpha\right)$ and a continuous nondecreasing function $\vartheta:[0, \infty) \rightarrow(0, \infty)$ such that for any $(t, x) \in J \times C_{\alpha}$, we have $E\|F(t, x(t))\|^{2} \leq N(t) \times \vartheta\left(\|x\|_{C_{\alpha}}\right)$, $\liminf _{r \rightarrow \infty} \frac{\vartheta(r)}{r} d s=\Theta<\infty$

$\left(\mathrm{H}_{3}\right) \quad$ (3a) $h(t, \cdot): X \rightarrow X$ is continuous for each $t \in J$, and for each $x \in X$, the function $h(\cdot, x): J \rightarrow X$ is strongly measurable; 
(3b) there exist constants $\beta \in(0,1)$ and $L>0$ such that $h \in D\left(A^{\beta}\right)$ and for any $x, y \in C_{\alpha}(J, X), t \in J$, the function $A^{\beta} h(\cdot, x)$ is strongly measurable, and $A^{\beta} h(t, x(t))$ satisfies

$$
E\left\|A^{\beta} h(t, x(t))-A^{\beta} h(t, y(t))\right\|^{2} \leq L\|x-y\|_{C_{\alpha}},
$$

for all $x, y \in C_{\alpha}(J, X), t \in J$;

(3c) there exist a continuous nondecreasing function $\zeta:[0, \infty) \rightarrow(0, \infty)$ and a constant $r>0$ such that for a.e. $t \in J, x \in C_{\alpha}(J, X)$, we have

$$
E\left\|A^{\beta} h(t, x(t))\right\|^{2} \leq L\left(1+\zeta\left(\|x\|_{C_{\alpha}}\right)\right), \quad \liminf _{r \rightarrow \infty} \frac{\zeta(r)}{r} d s=\Pi_{1}<\infty
$$

$\left(\mathrm{H}_{4}\right) g: C_{\alpha}(J, X) \rightarrow \mathcal{L}_{0}^{2}(\Omega, X)$ is such that

(i) there exist a continuous nondecreasing function $\mu:[0, \infty) \rightarrow(0, \infty)$ such that $E\|g(x)\|^{2} \leq \mu\left(\|x\|_{C_{\alpha}}\right)$ for all $x \in C_{\alpha}(J, X)$ and a constant $r>0$ such that for a.e. $t \in J, x \in C_{\alpha}(J, X)$, we have

$$
E\|g(x)\|^{2} \leq L\left(1+\mu\left(\|x\|_{C_{\alpha}}\right)\right), \quad \liminf _{r \rightarrow \infty} \frac{\mu(r)}{r} d s=\Pi_{2}<\infty ;
$$

(ii) $g$ is a completely continuous map;

$\left(\mathrm{H}_{5}\right)$ the function $\sigma: J \rightarrow \mathcal{L}_{2}^{0}(X, Y)$ satisfies

$$
\int_{0}^{b}\|\sigma(s)\|_{\mathcal{L}_{2}^{0}}^{\frac{2}{2 \alpha_{1}-1}} d s<\infty, \quad \forall b>0
$$

We define the operator $\Psi: C_{\alpha}(J, X) \rightarrow C_{\alpha}(J, X)$ as follows:

$$
\begin{aligned}
(\Psi x)(t)= & t^{\alpha-1} \mathcal{P}_{\alpha}(t)\left[x_{0}+h(0, x(0))+g(x)\right]+h(t,(t)) \\
& +\int_{0}^{t}(t-s)^{\alpha-1} A \mathcal{P}_{\alpha}(t-s) h(s, x(s)) d s \\
& +\int_{0}^{t}(t-s)^{\alpha-1} \mathcal{P}_{\alpha}(t-s) F(s, x(s)) d \omega(s) \\
& +\int_{0}^{t}(t-s)^{\alpha-1} \mathcal{P}_{\alpha}(t-s) \sigma(s) d B_{Q}^{H}(s), \quad t \in J^{\prime} .
\end{aligned}
$$

Set

$$
\begin{aligned}
\left(\Psi_{1} x\right)(t)= & t^{\alpha-1} \mathcal{P}_{\alpha}(t)\left[x_{0}+h(0, x(0))+g(x)\right]+h(t, x(t)) \\
& +\int_{0}^{t}(t-s)^{\alpha-1} A \mathcal{P}_{\alpha}(t-s) h(s, x(s)) d s, \quad t \in J^{\prime}, \\
\left(\Psi_{2} x\right)(t)= & \int_{0}^{t}(t-s)^{\alpha-1} \mathcal{P}_{\alpha}(t-s) F(s, x(s)) d \omega(s) \\
& +\int_{0}^{t}(t-s)^{\alpha-1} \mathcal{P}_{\alpha}(t-s) \sigma(s) d B_{Q}^{H}(s), \quad t \in J^{\prime} .
\end{aligned}
$$


According to assumption $\left(\mathrm{H}_{3}\right)$ and Lemma 2.3, we obtain

$$
\begin{aligned}
E\left\|\int_{0}^{t}(t-s)^{\alpha-1} A \mathcal{P}_{\alpha}(t-s) h(s, x(s)) d s\right\|^{2} \\
\leq E \int_{0}^{t}\left\|(t-s)^{\alpha-1} A^{1-\beta} \mathcal{P}_{\alpha}(t-s) A^{\beta} h(s, x(s))\right\|^{2} d s \\
\leq \int_{0}^{t}\left\|(t-s)^{\alpha-1} A^{1-\beta} \mathcal{P}_{\alpha}(t-s)\right\| d s \\
\quad \times \int_{0}^{t}(t-s)^{\alpha-1} A^{1-\beta} \mathcal{P}_{\alpha}(t-s) E\left\|A^{\beta} h(s, x(s))\right\|^{2} d s \\
\leq \frac{\alpha^{2}\left(C_{1-\beta}\right)^{2} \Gamma^{2}(1+\beta)}{\Gamma^{2}(1+\alpha \beta)} \\
\quad \times \int_{0}^{t}(t-s)^{\alpha \beta-1} d s \int_{0}^{t}(t-s)^{\alpha \beta-1} E\left\|A^{\beta} h(s, x(s))\right\|^{2} d s \\
\leq b^{2 \alpha \beta} \frac{\left(C_{1-\beta}\right)^{2} \Gamma^{2}(1+\beta)}{\beta^{2} \Gamma^{2}(1+\alpha \beta)} L\left(1+\zeta\left(\|x\|_{C_{\alpha}}\right)\right) \\
=b^{2 \alpha \beta} K(\alpha, \beta) L\left(1+\zeta\left(\|x\|_{C_{\alpha}}\right)\right) .
\end{aligned}
$$

Now, from assumption $\left(\mathrm{H}_{2}\right)$, we get

$$
\begin{aligned}
& E\left\|\int_{0}^{t}(t-s)^{\alpha-1} \mathcal{P}_{\alpha}(t-s) F(s, x(s)) d \omega(s)\right\|^{2} \\
& \quad \leq \operatorname{Tr} Q \frac{M^{2}}{\Gamma^{2}(\alpha)} \int_{0}^{t}(t-s)^{2(\alpha-1)} E\|F(s, x(s))\|^{2} d s \\
& \quad \leq \operatorname{Tr} Q \frac{M^{2}}{\Gamma^{2}(\alpha)}\left(\int_{0}^{t}(t-s)^{\frac{2(\alpha-1)}{2-2 \alpha_{1}}} d s\right)^{2-2 \alpha_{1}} \vartheta\left(\|x\|_{C_{\alpha}}\right)\|N\|_{L^{\frac{1}{2 \alpha_{1}-1}}} \\
& \quad \leq \operatorname{Tr} Q \frac{M^{2}}{\Gamma^{2}(\alpha)} \vartheta\left(\|x\|_{C_{\alpha}}\right) \frac{b^{(1+c)\left(2-2 \alpha_{1}\right)}}{(1+c)^{2-2 \alpha_{1}}}\|N\|_{L^{2 \alpha_{1}-1}} \\
& \quad=\operatorname{Tr} Q \frac{M^{2}}{\Gamma^{2}(\alpha)} \vartheta\left(\|x\|_{C_{\alpha}}\right) \Lambda\|N\|_{L^{\frac{1}{2 \alpha_{1}-1}}} \cdot
\end{aligned}
$$

For the last term of the mild solution, from assumption $\left(\mathrm{H}_{5}\right)$, we have the estimate

$$
\begin{aligned}
E \| & \int_{0}^{t}(t-s)^{\alpha-1} \mathcal{P}_{\alpha}(t-s) \sigma(s) d B_{Q}^{H}(s) \|^{2} \\
\leq & c_{0} H(2 H-1) t^{2 H-1} \frac{M^{2}}{\Gamma^{2}(\alpha)} \int_{0}^{t}(t-s)^{2(\alpha-1)}\|\sigma(s)\|_{\mathcal{L}_{2}^{0}}^{2} d s \\
\leq & c_{0} H(2 H-1) t^{2 H-1} \frac{M^{2}}{\Gamma^{2}(\alpha)}\left(\int_{0}^{t}(t-s)^{\frac{2(\alpha-1)}{2-2 \alpha_{1}}} d s\right)^{2-2 \alpha_{1}} \\
& \times\left(\int_{0}^{b}\|\sigma(s)\|^{\frac{2}{2 \alpha_{1}-1}} d s\right)^{2 \alpha_{1}-1} \\
\leq & c_{0} H(2 H-1) \frac{M^{2}}{\Gamma^{2}(\alpha)} \frac{b^{(1+c)\left(2-2 \alpha_{1}\right)+2 H-1}}{(1+c)^{2-2 \alpha_{1}}}\left(\int_{0}^{b}\|\sigma(s)\|^{\frac{2}{2 \alpha_{1}-1}} d s\right)^{2 \alpha_{1}-1} .
\end{aligned}
$$

In the following, we set to present our first existence result for system (1.1). 
Theorem 3.1 Suppose that hypotheses $\left(\mathrm{H}_{1}\right)-\left(\mathrm{H}_{5}\right)$ hold, then system (1.1) has at least one mild solution defined on $J^{\prime}$ provided that $2 b^{2(1-\alpha)} M_{0} L+2 b^{2(1-\alpha+\alpha \beta)} K^{2}(\alpha, \beta) L<1$ and

$$
\begin{aligned}
& \frac{15 M^{2}}{\Gamma^{2}(\alpha)}\left[M_{0}^{2} L \Pi_{1}+L \Pi_{2}\right]+5 b^{2(1-\alpha)} M_{0} L \Pi_{1}+5 b^{2(1-\alpha+\alpha \beta)} K^{2}(\alpha, \beta) L \Pi_{1} \\
& \quad+5 b^{2(1-\alpha)} \frac{M^{2}}{\Gamma^{2}(\alpha)} \Theta \Lambda\|N\|_{\frac{1}{2 \alpha_{1}-1}}<1 .
\end{aligned}
$$

Proof Denote $B_{q}=\left\{x \in C_{\alpha}(J, X),\|x\|_{C_{\alpha}} \leq q\right\}$. Then it is obvious that $B_{q}$ is a bounded, closed, convex set in $C_{\alpha}(J, X)$. We demonstrate the proof in six steps.

Step 1. We shall show that there exists a constant $r=r(a)$ such that $\Psi\left(B_{r}\right) \subset B_{r}$.

In fact, if this claim is not true, then for each positive constant $r$ there exists some $x^{(r)} \in$ $B_{r}$ such that $\Psi\left(x^{(r)}\right) \notin B_{r}$, i.e.,

$$
\begin{aligned}
& r<\left\|\Psi\left(x^{(r)}\right)\right\|_{C_{\alpha}}^{2} \\
& \leq \sup _{t \in J^{\prime}} t^{2(1-\alpha)}\left\{5 E\left\|t^{\alpha-1} \mathcal{P}_{\alpha}(t)\left[x_{0}+h\left(0, x^{(r)}(0)\right)+g\left(x^{(r)}\right)\right]\right\|^{2}\right. \\
& +5 E\left\|h\left(t, x^{(r)}(t)\right)\right\|^{2} \\
& +5 E\left\|\int_{0}^{t}(t-s)^{\alpha-1} A \mathcal{P}_{\alpha}(t-s) h\left(s, x^{(r)}(s)\right) d s\right\|^{2} \\
& +5 E\left\|\int_{0}^{t}(t-s)^{\alpha-1} \mathcal{P}_{\alpha}(t-s) F\left(s, x^{(r)}(s)\right) d \omega(s)\right\|^{2} \\
& \left.+5 E\left\|\int_{0}^{t}(t-s)^{\alpha-1} \mathcal{P}_{\alpha}(t-s) \sigma(s) d B_{Q}^{H}(s)\right\|^{2}\right\} \\
& \leq \frac{5 M^{2}}{\Gamma^{2}(\alpha)}\left[E\left\|x_{0}+h\left(0, x^{(r)}(0)\right)+g\left(x^{(r)}\right)\right\|^{2}\right] \\
& +5 b^{2(1-\alpha)}\left\|A^{-\beta}\right\|^{2} L\left(1+\zeta\left\|x^{(r)}\right\|_{C_{\alpha}}\right) \\
& +5 b^{2(1-\alpha+\alpha \beta)} K^{2}(\alpha, \beta) L\left(1+\zeta\left\|x^{(r)}\right\|_{C_{\alpha}}\right) \\
& +5 b^{2(1-\alpha)} \operatorname{Tr} Q \frac{M^{2}}{\Gamma^{2}(\alpha)} \vartheta\left(\left\|x^{(r)}\right\|_{C_{\alpha}}\right) \Lambda\|N\|_{L^{\frac{1}{2 \alpha_{1}-1}}} \\
& +5 c_{0} H(2 H-1) \frac{M^{2}}{\Gamma^{2}(\alpha)} \frac{b^{(1+c)\left(2-2 \alpha_{1}\right)+2 H+1-2 \alpha}}{(1+c)^{2-2 \alpha_{1}}}\left(\int_{0}^{b}\|\sigma(s)\|^{\frac{2}{2 \alpha_{1}-1}} d s\right)^{2 \alpha_{1}-1} \\
& \leq \frac{5 M^{2}}{\Gamma^{2}(\alpha)}\left[3 E\left\|x_{0}\right\|^{2}+3 M_{0}^{2} L(1+\zeta(r))+3 L(1+\mu(r))\right] \\
& +5 b^{2(1-\alpha)} M_{0}^{2} L(1+\zeta(r))+5 b^{2(1-\alpha+\alpha \beta)} K^{2}(\alpha, \beta) L(1+\zeta(r)) \\
& +5 b^{2(1-\alpha)} \operatorname{Tr} Q \frac{M^{2}}{\Gamma^{2}(\alpha)} \vartheta(r) \Lambda\|N\|_{L^{2 \alpha_{1}-1}} \\
& +5 c_{0} H(2 H-1) \frac{M^{2}}{\Gamma^{2}(\alpha)} \frac{b^{(1+c)\left(2-2 \alpha_{1}\right)+2 H+1-2 \alpha}}{(1+c)^{2-2 \alpha_{1}}}\left(\int_{0}^{b}\|\sigma(s)\|^{\frac{2}{2 \alpha_{1}-1}} d s\right)^{2 \alpha_{1}-1} \text {. }
\end{aligned}
$$


Dividing both sides by $r$ and letting $r \rightarrow \infty$ yields

$$
\begin{aligned}
& \frac{15 M^{2}}{\Gamma^{2}(\alpha)}\left[M_{0}^{2} L \Pi_{1}+L \Pi_{2}\right]+5 b^{2(1-\alpha)} M_{0} L \Pi_{1}+5 b^{2(1-\alpha+\alpha \beta)} K^{2}(\alpha, \beta) L \Pi_{1} \\
& \quad+5 b^{2(1-\alpha)} \frac{M^{2}}{\Gamma^{2}(\alpha)} \Theta \Lambda\|N\|_{\frac{1}{2 \alpha_{1}-1}}>1 .
\end{aligned}
$$

This contradicts the assumption of Theorem 3.1, which implies that there exists $r$ such that $\Psi$ maps $B_{r}$ into itself.

Step 2. We show that $\Psi_{1}$ is a contraction on $B_{r}$.

For any $x, y \in B_{r}$, we derive

$$
\begin{aligned}
\|( & \left.\Psi_{1} x\right)(t)-\left(\Psi_{1} y\right)(t) \|_{C_{\alpha}}^{2} \\
= & \sup _{t \in J^{\prime}} t^{2(1-\alpha)} E\left\|\left(\Psi_{1} x\right)(t)-\left(\Psi_{1} y\right)(t)\right\|^{2} \\
\leq & \sup _{t \in J^{\prime}} 2 t^{2(1-\alpha)} E\|h(t, x(t))-h(t, y(t))\|^{2} \\
& +\sup _{t \in J^{\prime}} 2 t^{2(1-\alpha)} E\left\|\int_{0}^{t}(t-s)^{\alpha-1} A \mathcal{P}_{\alpha}(t-s)[h(s, x(s))-h(s, y(s))] d s\right\|^{2} \\
\leq & \sup _{t \in J^{\prime}} 2 t^{2(1-\alpha)}\left\|A^{-\beta}\right\|^{2} E\left\|A^{\beta} h(t, x(t))-A^{\beta} h(t, y(t))\right\|^{2} \\
& +\sup _{t \in J^{\prime}} 2 t^{2(1-\alpha)} \int_{0}^{t}(t-s)^{\alpha-1} A^{1-\beta} \mathcal{P}_{\alpha}(t-s) d s \\
& \times \int_{0}^{t}(t-s)^{\alpha-1} A^{1-\beta} \mathcal{P}_{\alpha}(t-s) E\left\|A^{\beta} h(s, x(s))-A^{\beta} h(s, y(s))\right\|^{2} d s \\
\leq & 2 b^{2(1-\alpha)} M_{0}^{2} L\|x-y\|_{C_{1-\alpha}}+2 b^{2(1-\alpha+\alpha \beta)} K^{2}(\alpha, \beta) L\|x-y\|_{C_{\alpha}} \\
= & {\left[2 b^{2(1-\alpha)} M_{0}^{2} L+2 b^{2(1-\alpha+\alpha \beta)} K^{2}(\alpha, \beta) L\right]\|x-y\|_{C_{\alpha}} . }
\end{aligned}
$$

Thus, $\Psi_{1}$ is a contraction by assumption of Theorem 3.1 .

Step 3. $\Psi_{2}$ is completely continuous.

To prove this assertion, we subdivide Step 3 into three claims.

Claim 1. $\Psi_{2}$ maps bounded sets into uniformly bounded sets in $C_{\alpha}(J, X)$.

We only need to show that there exists constant $\Delta>0$ such that for each $\Psi_{2} x, x \in B_{r}$, $\left\|\Psi_{2} x\right\|_{C_{\alpha}} \leq \Delta$ holds. In fact, for each $t \in J^{\prime}$, by using Hölder's inequality, we have

$$
\begin{aligned}
\left\|\Psi_{2} x\right\|_{C_{\alpha}}^{2}= & \sup _{t \in J^{\prime}} t^{2(1-\alpha)} E \| t^{\alpha-1} \mathcal{P}_{\alpha}(t)\left[x_{0}+h(0, x(0))+g(x)\right] \\
& +\int_{0}^{t}(t-s)^{\alpha-1} \mathcal{P}_{\alpha}(t-s) F(s, x(s)) d \omega(s) \\
& +\int_{0}^{t}(t-s)^{\alpha-1} \mathcal{P}_{\alpha}(t-s) \sigma(s) d B_{Q}^{H} \|^{2} \\
\leq & 3 \frac{M^{2}}{\Gamma^{2}(\alpha)} E\left\|x_{0}+h(0, x(0))+g(x)\right\|^{2} \\
& +3 b^{2(1-\alpha)} \operatorname{Tr} Q \frac{M^{2}}{\Gamma^{2}(\alpha)} \vartheta(r) \Lambda\|N\|_{L^{\frac{1}{2 \alpha 1}-1}}
\end{aligned}
$$




$$
\begin{aligned}
& +3 b^{2 H+1-2 \alpha} c_{0} H(2 H-1) \frac{M^{2}}{\Gamma^{2}(\alpha)} \frac{b^{(1+c)\left(2-2 \alpha_{1}\right)}}{(1+c)^{2-2 \alpha_{1}}}\left(\int_{0}^{b}\|\sigma(s)\|^{\frac{2}{2 \alpha_{1}-1}} d s\right)^{2 \alpha_{1}-1} \\
\leq & 3 \frac{M^{2}}{\Gamma^{2}(\alpha)}\left[3 E\left\|x_{0}\right\|^{2}+3 M_{0}^{2} L(1+\zeta(r))+3 L(1+\mu(r))\right] \\
& +3 b^{2-2 \alpha} \operatorname{Tr} Q \frac{M^{2}}{\Gamma^{2}(\alpha)} \vartheta(r) \Lambda\|N\| \frac{1}{L^{2 \alpha_{1}-1}} \\
& +3 b^{2 H+1-2 \alpha} c_{0} H(2 H-1) \frac{M^{2}}{\Gamma^{2}(\alpha)} \frac{b^{(1+c)\left(2-2 \alpha_{1}\right)}}{(1+c)^{2-2 \alpha_{1}}}\left(\int_{0}^{b}\|\sigma(s)\|^{\frac{2}{2 \alpha_{1}-1}} d s\right)^{2 \alpha_{1}-1}
\end{aligned}
$$

$:=\Delta$.

Consequently, for each $\rho \in \Psi_{2} x$, we have $\|\rho(t)\|_{C_{\alpha}} \leq \Delta$.

Claim 2. $\Psi_{2}\left(B_{r}\right)$ is equicontinuous on $B_{r}$.

Denoting $\bar{E}=\left\{y \in C(J, X): y(t)=t^{1-\alpha}\left(\Psi_{2} x\right)(t), y(0)=y\left(0^{+}\right), x \in B_{r}\right\}$, for $t_{1}=0,0<t_{2} \leq b$, we can obtain

$$
\begin{aligned}
& E\left\|y\left(t_{2}\right)-y(0)\right\|^{2} \\
& \leq 3\left\|\left[\mathcal{P}_{\alpha}\left(t_{2}\right)-\mathcal{P}_{\alpha}(0)\right]\left[x_{0}+h(0, x(0))+g(x)\right]\right\|^{2} \\
& \quad+3 t_{2}^{1-\alpha}\left\|\int_{0}^{t_{2}}\left(t_{2}-s\right)^{\alpha-1} \mathcal{P}_{\alpha}\left(t_{2}-s\right) F(s, x(s)) d \omega(s)\right\|^{2} \\
& \quad+3 t_{2}^{1-\alpha}\left\|\int_{0}^{t_{2}}\left(t_{2}-s\right)^{\alpha-1} \mathcal{P}_{\alpha}\left(t_{2}-s\right) \sigma(s) d B_{Q}^{H}(s)\right\|^{2} \rightarrow 0, \quad \text { as } t_{2} \rightarrow t_{1}=0 .
\end{aligned}
$$

For $0<t_{1}<t_{2} \leq b$, the strong continuity of $\left\{\mathcal{P}_{\alpha}(t): t \geq 0\right\}$ implies that there exists a constant $\delta>0$ such that $\left|t_{2}-t_{1}\right|<\delta$ and $\left\|\mathcal{P}_{\alpha}\left(t_{1}\right)-\mathcal{P}_{\alpha}\left(t_{2}\right)\right\|<\tau$. In addition, note that $c=$ $\frac{\alpha-1}{1-\alpha_{1}}$, then for $\forall x \in B_{r}$, this yields that

$$
\begin{aligned}
& E\left\|y\left(t_{2}\right)-y\left(t_{1}\right)\right\|^{2} \\
& \leq 9 E\left\|\mathcal{P}_{\alpha}\left(t_{2}\right)\left[x_{0}+h(0, x(0))+g(x)\right]-\mathcal{P}_{\alpha}\left(t_{1}\right)\left[x_{0}+h(0, x(0))+g(x)\right]\right\|^{2} \\
& \quad+9 E\left\|\int_{0}^{t_{1}}\left[t_{2}^{1-\alpha}\left(t_{2}-s\right)^{\alpha-1}-t_{1}^{1-\alpha}\left(t_{1}-s\right)^{\alpha-1}\right] \mathcal{P}_{\alpha}\left(t_{2}-s\right) F(s, x(s)) d \omega(s)\right\|^{2} \\
& \quad+9 E\left\|\int_{0}^{t_{1}}\left[t_{2}^{1-\alpha}\left(t_{2}-s\right)^{\alpha-1}-t_{1}^{1-\alpha}\left(t_{1}-s\right)^{\alpha-1}\right] \mathcal{P}_{\alpha}\left(t_{2}-s\right) \sigma(s) d B_{Q}^{H}(s)\right\|^{2} \\
& \quad+9 E\left\|\int_{0}^{t_{1}-\varepsilon} t_{1}^{1-\alpha}\left(t_{1}-s\right)^{\alpha-1}\left[\mathcal{P}_{\alpha}\left(t_{2}-s\right)-\mathcal{P}_{\alpha}\left(t_{1}-s\right)\right] F(s, x(s)) d \omega(s)\right\|^{2} \\
& \quad+9 E\left\|\int_{0}^{t_{1}-\varepsilon} t_{1}^{1-\alpha}\left(t_{1}-s\right)^{\alpha-1}\left[\mathcal{P}_{\alpha}\left(t_{2}-s\right)-\mathcal{P}_{\alpha}\left(t_{1}-s\right)\right] \sigma(s) d B_{Q}^{H}(s)\right\|^{2} \\
& \quad+9 E\left\|\int_{t_{1}-\varepsilon}^{t_{1}} t_{1}^{1-\alpha}\left(t_{1}-s\right)^{\alpha-1}\left[\mathcal{P}_{\alpha}\left(t_{2}-s\right)-\mathcal{P}_{\alpha}\left(t_{1}-s\right)\right] F(s, x(s)) d \omega(s)\right\|^{2} \\
& \quad+9 E\left\|\int_{t_{1}-\varepsilon}^{t_{1}} t_{1}^{1-\alpha}\left(t_{1}-s\right)^{\alpha-1}\left[\mathcal{P}_{\alpha}\left(t_{2}-s\right)-\mathcal{P}_{\alpha}\left(t_{1}-s\right)\right] \sigma(s) d B_{Q}^{H}(s)\right\|^{2} \\
& \quad+9 E\left\|\int_{t_{1}}^{t_{2}} t_{2}^{1-\alpha}\left(t_{2}-s\right)^{\alpha-1} \mathcal{P}_{\alpha}\left(t_{2}-s\right) F(s, x(s)) d \omega(s)\right\| \|^{2}
\end{aligned}
$$




$$
\begin{aligned}
& +9 E\left\|\int_{t_{1}}^{t_{2}} t_{2}^{1-\alpha}\left(t_{2}-s\right)^{\alpha-1} \mathcal{P}_{\alpha}\left(t_{2}-s\right) \sigma(s) d B_{Q}^{H}(s)\right\|^{2} \\
& \leq 9\left\|\mathcal{P}_{\alpha}\left(t_{2}\right)-\mathcal{P}_{\alpha}\left(t_{1}\right)\right\|^{2} E\left\|x_{0}+h(0, x(0))+g(x)\right\|^{2} \\
& +9 \operatorname{Tr} Q \int_{0}^{t_{1}}\left\|t_{2}^{1-\alpha}\left(t_{2}-s\right)^{\alpha-1}-t_{1}^{1-\alpha}\left(t_{1}-s\right)^{\alpha-1}\right\|^{2} \\
& \times\left\|\mathcal{P}_{\alpha}\left(t_{2}-s\right)\right\|^{2} E\|F(s, x(s))\|^{2} d s \\
& +9 c_{0} H(2 H-1) t_{1}^{2 H-1} \int_{0}^{t_{1}}\left\|t_{2}^{1-\alpha}\left(t_{2}-s\right)^{\alpha-1}-t_{1}^{1-\alpha}\left(t_{1}-s\right)^{\alpha-1}\right\|^{2} \\
& \times\left\|\mathcal{P}_{\alpha}\left(t_{2}-s\right)\right\|^{2}\|\sigma(s)\|_{\mathcal{L}_{0}^{2}}^{2} d s \\
& +9 \sup _{\left[0, t_{1}-\varepsilon\right]}\left\|\mathcal{P}_{\alpha}\left(t_{2}-s\right)-\mathcal{P}_{\alpha}\left(t_{1}-s\right)\right\|^{2} \operatorname{Tr} Q \vartheta(r)\|N\|_{L^{\frac{1}{2 \alpha_{1}-1}}} \\
& \times\left[\frac{t_{1}^{(1+c)\left(2-2 \alpha_{1}\right)}}{(1+c)^{2-2 \alpha_{1}}}-\frac{\varepsilon^{(1+c)\left(2-2 \alpha_{1}\right)}}{(1+c)^{2-2 \alpha_{1}}}\right] \\
& +9 c_{0} H(2 H-1) b^{2 H-1} \sup _{\left[0, t_{1}-\varepsilon\right]}\left\|\mathcal{P}_{\alpha}\left(t_{2}-s\right)-\mathcal{P}_{\alpha}\left(t_{1}-s\right)\right\|^{2} \\
& \times\left[\frac{t_{1}^{(1+c)\left(2-2 \alpha_{1}\right)}}{(1+c)^{2-2 \alpha_{1}}}-\frac{\varepsilon^{(1+c)\left(2-2 \alpha_{1}\right)}}{(1+c)^{2-2 \alpha_{1}}}\right]\left(\int_{0}^{b}\|\sigma(s)\|^{\frac{2}{2 \alpha_{1}-1}} d s\right)^{2 \alpha_{1}-1} \\
& +9 \operatorname{Tr} Q \frac{4 M^{2}}{\Gamma^{2}(\alpha)} \int_{t_{1}-\varepsilon}^{t_{1}} t_{1}^{2(1-\alpha)}\left(t_{1}-s\right)^{2(\alpha-1)} E\|F(s, x(s))\|^{2} d s \\
& +9 c_{0} H(2 H-1) t_{1}^{2 H-1} \frac{4 M^{2}}{\Gamma^{2}(\alpha)} \int_{t_{1}-\varepsilon}^{t_{1}} t_{1}^{2(1-\alpha)}\left(t_{1}-s\right)^{2(\alpha-1)}\|\sigma(s)\|_{\mathcal{L}_{2}^{0}}^{2} d s \\
& +9 \operatorname{Tr} Q \frac{M^{2}}{\Gamma^{2}(\alpha)} b^{2-2 \alpha} \int_{t_{1}}^{t_{2}}\left(t_{2}-s\right)^{2(\alpha-1)} E\|F(s, x(s))\|^{2} d s \\
& +9 c_{0} H(2 H-1) \frac{M^{2}}{\Gamma^{2}(\alpha)} b^{2 H+1-2 \alpha} \int_{t_{1}}^{t_{2}}\left(t_{2}-s\right)^{2(\alpha-1)}\|\sigma(s)\|_{\mathcal{L}_{2}^{0}}^{2} d s \\
& =9 \sum_{i=1}^{9} I_{i}
\end{aligned}
$$

where

$$
\begin{aligned}
I_{1}= & \left\|\mathcal{P}_{\alpha}\left(t_{2}\right)-\mathcal{P}_{\alpha}\left(t_{1}\right)\right\|^{2} E\left\|x_{0}+h(0, x(0))+g(x)\right\|^{2}, \\
I_{2}= & \operatorname{Tr} Q \int_{0}^{t_{1}}\left\|t_{2}^{1-\alpha}\left(t_{2}-s\right)^{\alpha-1}-t_{1}^{1-\alpha}\left(t_{1}-s\right)^{\alpha-1}\right\|^{2} \\
& \times\left\|\mathcal{P}_{\alpha}\left(t_{2}-s\right)\right\|^{2} E\|F(s, x(s))\|^{2} d s, \\
I_{3}= & c_{0} H(2 H-1) t_{1}^{2 H-1} \int_{0}^{t_{1}}\left\|t_{2}^{1-\alpha}\left(t_{2}-s\right)^{\alpha-1}-t_{1}^{1-\alpha}\left(t_{1}-s\right)^{\alpha-1}\right\|^{2} \\
& \times\left\|\mathcal{P}_{\alpha}\left(t_{2}-s\right)\right\|^{2}\|\sigma(s)\|^{2} d s, \\
I_{4}= & \sup _{\left[0, t_{1}-\varepsilon\right]}\left\|\mathcal{P}_{\alpha}\left(t_{2}-s\right)-\mathcal{P}_{\alpha}\left(t_{1}-s\right)\right\|^{2} \operatorname{Tr} Q \vartheta(r)\|N\|_{L^{2 \alpha_{1}-1}} \\
& \times\left[\frac{t_{1}^{(1+c)\left(2-2 \alpha_{1}\right)}}{(1+c)^{2-2 \alpha_{1}}}-\frac{\varepsilon^{(1+c)\left(2-2 \alpha_{1}\right)}}{(1+c)^{2-2 \alpha_{1}}}\right],
\end{aligned}
$$




$$
\begin{aligned}
& I_{5}=c_{0} H(2 H-1) b^{2 H-1} \sup _{\left[0, t_{1}-\varepsilon\right]}\left\|\mathcal{P}_{\alpha}\left(t_{2}-s\right)-\mathcal{P}_{\alpha}\left(t_{1}-s\right)\right\|^{2} \\
& \times\left[\frac{t_{1}^{(1+c)\left(2-2 \alpha_{1}\right)}}{(1+c)^{2-2 \alpha_{1}}}-\frac{\varepsilon^{(1+c)\left(2-2 \alpha_{1}\right)}}{(1+c)^{2-2 \alpha_{1}}}\right]\left(\int_{0}^{b}\|\sigma(s)\|^{\frac{2}{2 \alpha_{1}-1}} d s\right)^{2 \alpha_{1}-1}, \\
& I_{6}=\operatorname{Tr} Q \frac{4 M^{2}}{\Gamma^{2}(\alpha)} \int_{t_{1}-\varepsilon}^{t_{1}} t_{1}^{2(1-\alpha)}\left(t_{1}-s\right)^{2(\alpha-1)} E\|F(s, x(s))\|^{2} d s \\
& \leq \operatorname{Tr} Q \frac{4 M^{2}}{\Gamma^{2}(\alpha)} t_{1}^{2-2 \alpha} \frac{\varepsilon^{(1+c)\left(2-2 \alpha_{1}\right)}}{(1+c)^{2-2 \alpha_{1}}} \vartheta(r)\|N\|_{L^{2 \alpha_{1}-1}}, \\
& I_{7}=c_{0} H(2 H-1) t_{1}^{2 H-1} \frac{4 M^{2}}{\Gamma^{2}(\alpha)} \int_{t_{1}-\varepsilon}^{t_{1}} t_{1}^{2(1-\alpha)}\left(t_{1}-s\right)^{2(\alpha-1)}\|\sigma(s)\|_{\mathcal{L}_{2}^{0}}^{2} d s \\
& \leq c_{0} H(2 H-1) t_{1}^{2 H+1-2 \alpha} \frac{4 M^{2}}{\Gamma^{2}(\alpha)} \frac{\varepsilon^{(1+c)\left(2-2 \alpha_{1}\right)}}{(1+c)^{2-2 \alpha_{1}}}\left(\int_{0}^{b}\|\sigma(s)\|^{2 \alpha_{1}-1} d s\right)^{2 \alpha_{1}-1}, \\
& I_{8}=\operatorname{Tr} Q \frac{M^{2}}{\Gamma^{2}(\alpha)} b^{2-2 \alpha} \int_{t_{1}}^{t_{2}}\left(t_{2}-s\right)^{2(\alpha-1)} E\|F(s, x(s))\|^{2} d s \\
& \leq \operatorname{Tr} Q \frac{M^{2}}{\Gamma^{2}(\alpha)} b^{2-2 \alpha} \frac{\left(t_{2}-t_{1}\right)^{(1+c)\left(2-2 \alpha_{1}\right)}}{(1+c)^{2-2 \alpha_{1}}} \vartheta(r)\|N\|_{L^{2 \alpha_{1}-1}}, \\
& I_{9}=c_{0} H(2 H-1) \frac{M^{2}}{\Gamma^{2}(\alpha)} b^{2 H+1-2 \alpha} \int_{t_{1}}^{t_{2}}\left(t_{2}-s\right)^{2(\alpha-1)}\|\sigma(s)\|_{\mathcal{L}_{2}^{0}}^{2} d s \\
& \leq c_{0} H(2 H-1) \frac{M^{2}}{\Gamma^{2}(\alpha)} b^{2 H+1-2 \alpha} \frac{\left(t_{2}-t_{1}\right)^{(1+c)\left(2-2 \alpha_{1}\right)}}{(1+c)^{2-2 \alpha_{1}}}\left(\int_{0}^{b}\|\sigma(s)\|^{\frac{2}{2 \alpha_{1}-1}} d s\right)^{2 \alpha_{1}-1} .
\end{aligned}
$$

Since $p \in(0, \alpha)$, we have $(1+c)\left(2-2 \alpha_{1}\right)>0$, thus the terms from $I_{6}$ to $I_{9}$ tend to zero as $t_{2}-t_{1} \rightarrow 0$ and $\varepsilon \rightarrow 0$. The strong continuity of $\left\{\mathcal{P}_{\alpha}(t): t \geq 0\right\}$ indicates that $\| \mathcal{P}_{\alpha}\left(t_{2}-s\right)-$ $\mathcal{P}_{\alpha}\left(t_{1}-s\right) \|^{2} \rightarrow 0$ as $\delta \rightarrow 0$. Hence $I_{1}, I_{4}, I_{5}$ also tend to zero as $t_{2}-t_{1} \rightarrow 0$.

For $I_{2}$, by a standard calculation and for $p \in(0, \alpha)$, we have

$$
\begin{aligned}
I_{2} \leq & \operatorname{Tr} Q \int_{0}^{t_{1}}\left\|t_{2}^{1-\alpha}\left(t_{2}-s\right)^{\alpha-1}-t_{1}^{1-\alpha}\left(t_{1}-s\right)^{\alpha-1}\right\|^{2} \\
& \times\left\|\mathcal{P}_{\alpha}\left(t_{2}-s\right)\right\|^{2} E\|F(s, x(s))\|^{2} d s \\
\leq & \operatorname{Tr} Q \frac{M^{2}}{\Gamma^{2}(\alpha)} \int_{0}^{t_{1}}\left\|t_{2}^{1-\alpha}\left(t_{2}-s\right)^{\alpha-1}-t_{1}^{1-\alpha}\left(t_{1}-s\right)^{\alpha-1}\right\|^{2} E\|F(s, x(s))\|^{2} d s \\
\leq & \operatorname{Tr} Q \frac{M^{2}}{\Gamma^{2}(\alpha)} \frac{1}{(1+c)^{2-2 \alpha_{1}}}\left[\left(t_{2}-t_{1}\right)^{(1+c)\left(2-2 \alpha_{1}\right)}\right. \\
& \left.+t_{1}^{(1+c)\left(2-2 \alpha_{1}\right)}-t_{2}^{(1+c)\left(2-2 \alpha_{1}\right)}\right] \times \vartheta\left(\|x\|_{C_{\alpha}}\right)\|N\|_{L^{\frac{1}{2 \alpha_{1}-1}}} .
\end{aligned}
$$

Thus $I_{2}$ tends to zero as $t_{2}-t_{1} \rightarrow 0$. Similarly, we can get that $I_{3}$ tends to zero as $t_{2}-t_{1} \rightarrow 0$.

Therefore, the relationship of $E$ and $\left\{\Psi_{2} x: x \in B_{r}\right\}$ implies that $\Psi_{2}$ is equicontinuous on $B_{r}$.

Claim 3. $V(t)=\left\{\left(\Psi_{2} x\right)(t), x \in B_{r}\right\}$ is a relatively compact in $X$.

Let $0<t \leq b$ be fixed. Then for $\forall \lambda \in(0, t)$ and $\forall \delta>0, x \in B_{r}$, define an operator

$$
\begin{aligned}
\left(\Psi_{2}^{\lambda, \delta} x\right)(t)= & t^{1-\alpha} \mathcal{P}_{\alpha}(t)\left[x_{0}+h(0, x(0))+g(x)\right] \\
& +\alpha \int_{0}^{t-\lambda} \int_{\delta}^{\infty} \theta(t-s)^{\alpha-1} \phi_{\alpha}(\theta) S\left((t-s)^{\alpha} \theta\right) F(s, x(s)) d \omega(s)
\end{aligned}
$$




$$
\begin{aligned}
& +\alpha \int_{0}^{t-\lambda} \int_{\delta}^{\infty} \theta(t-s)^{\alpha-1} \phi_{\alpha}(\theta) S\left((t-s)^{\alpha} \theta\right) \sigma(s) d B_{Q}^{H}(s) \\
= & t^{1-\alpha} \mathcal{P}_{\alpha}(t)\left[x_{0}+h(0, x(0))+g(x)\right] \\
& +\alpha S\left(\lambda^{\alpha} \theta\right) \int_{0}^{t-\lambda} \int_{\delta}^{\infty} \theta(t-s)^{\alpha-1} \phi_{\alpha}(\theta) S\left((t-s)^{\alpha} \theta-\lambda^{\alpha} \theta\right) F(s, x(s)) d \omega(s) \\
& +\alpha S\left(\lambda^{\alpha} \theta\right) \int_{0}^{t-\lambda} \int_{\delta}^{\infty} \theta(t-s)^{\alpha-1} \phi_{\alpha}(\theta) S\left((t-s)^{\alpha} \theta-\lambda^{\alpha} \theta\right) \sigma(s) d B_{Q}^{H}(s) .
\end{aligned}
$$

From the compactness of $S\left(\lambda^{\alpha} \delta\right), \lambda^{\alpha} \delta>0$, we obtain that for $\forall \lambda \in(0, t)$ and $\forall \delta>0$, the set $V^{\lambda, \delta}(t)=\left\{\left(\Psi_{2}^{\lambda, \delta} x\right)(t), x \in B_{r}\right\}$ is relatively compact in $X$.

Moreover, for each $x \in B_{r}$, we have

$$
\begin{aligned}
& \left\|\left(\Psi_{2} x\right)(t)-\left(\Psi_{2}^{\lambda, \delta} x\right)(t)\right\|_{C_{\alpha}} \\
& =\sup _{t \in J^{\prime}} t^{2(1-\alpha)} E \| \int_{0}^{t} \int_{0}^{\delta} \alpha \theta(t-s)^{\alpha-1} \phi_{\alpha}(\theta) S\left((t-s)^{\alpha} \theta\right) F(s, x(s) d \omega(s) \\
& +\int_{0}^{t} \int_{\delta}^{\infty} \alpha \theta(t-s)^{\alpha-1} \phi_{\alpha}(\theta) S\left((t-s)^{\alpha} \theta\right) F(s, x(s) d \omega(s) \\
& +\int_{0}^{t} \int_{\delta}^{\infty} \alpha \theta(t-s)^{\alpha-1} \phi_{\alpha}(\theta) S\left((t-s)^{\alpha} \theta\right) \sigma(s) d B_{Q}^{H}(s) \\
& -\int_{0}^{t-\lambda} \int_{\delta}^{\infty} \alpha \theta(t-s)^{\alpha-1} \phi_{\alpha}(\theta) S\left((t-s)^{\alpha} \theta\right) F(s, x(s) d \omega(s) \\
& -\int_{0}^{t-\lambda} \int_{\delta}^{\infty} \alpha \theta(t-s)^{\alpha-1} \phi_{\alpha}(\theta) S\left((t-s)^{\alpha} \theta\right) \sigma(s) d B_{Q}^{H}(s) \|^{2} \\
& \leq 4 \alpha^{2} \sup _{t \in J^{\prime}} t^{2(1-\alpha)} E \| \int_{0}^{t} \int_{0}^{\delta} \theta(t-s)^{\alpha-1} \phi_{\alpha}(\theta) S\left((t-s)^{\alpha} \theta\right) F\left(s, x(s) d \omega(s) \|^{2}\right. \\
& +4 \alpha^{2} \sup _{t \in J^{\prime}} t^{2(1-\alpha)} E\left\|\int_{0}^{t} \int_{0}^{\delta} \theta(t-s)^{\alpha-1} \phi_{\alpha}(\theta) S\left((t-s)^{\alpha} \theta\right) \sigma(s) d B_{Q}^{H}(s)\right\|^{2} \\
& +4 \alpha^{2} \sup _{t \in J^{\prime}} t^{2(1-\alpha)} E \| \int_{t-\lambda}^{t} \int_{\delta}^{\infty} \theta(t-s)^{\alpha-1} \phi_{\alpha}(\theta) S\left((t-s)^{\alpha} \theta\right) F\left(s, x(s) d \omega(s) d s \|^{2}\right. \\
& +4 \alpha^{2} \sup _{t \in J^{\prime}} t^{2(1-\alpha)} E\left\|\int_{t-\lambda}^{t} \int_{\delta}^{\infty} \theta(t-s)^{\alpha-1} \phi_{\alpha}(\theta) S\left((t-s)^{\alpha} \theta\right) \sigma(s) d B_{Q}^{H}(s)\right\|^{2} \\
& \leq 4 \alpha^{2} b^{2-2 \alpha} M^{2} \operatorname{Tr} Q \Lambda \vartheta(r)\|N\|_{L^{2 \alpha_{1}-1}}\left(\int_{0}^{\delta} \theta \phi_{\alpha}(\theta) d \theta\right)^{2} \\
& +4 \alpha^{2} c_{0} H(2 H-1) b^{2 H+1-2 \alpha} M^{2} \Lambda\left(\int_{0}^{\delta} \theta \phi_{\alpha}(\theta) d \theta\right)^{2} \\
& \times\left(\int_{0}^{t}\|\sigma(s)\|^{\frac{2}{2 \alpha_{1}-1}} d s\right)^{2 \alpha_{1}-1} \\
& +4 \alpha^{2} b^{2-2 \alpha} M^{2} \operatorname{Tr} Q \vartheta(r)\|N\|_{L^{\frac{1}{2 \alpha_{1}-1}}} \frac{1}{\Gamma^{2}(\alpha+1)} \frac{\lambda^{(1+c)\left(2-2 \alpha_{1}\right)}}{(1+c)^{2-2 \alpha_{1}}} \\
& +4 \alpha^{2} c_{0} H(2 H-1) b^{2 H+1-2 \alpha} M^{2} \frac{1}{\Gamma^{2}(\alpha+1)} \frac{\lambda^{(1+c)\left(2-2 \alpha_{1}\right)}}{(1+c)^{2-2 \alpha_{1}}} \\
& \times\left(\int_{0}^{\lambda}\|\sigma(s)\|^{\frac{2}{2 \alpha_{1}-1}} d s\right)^{2 \alpha_{1}-1},
\end{aligned}
$$


where we have used the equality

$$
\int_{0}^{\infty} \theta^{\xi} \phi_{\alpha}(\theta) d \theta=\int_{0}^{\infty} \frac{1}{\theta^{\alpha \xi}} \psi_{\alpha}(\theta) d \theta=\frac{\Gamma(1+\xi)}{\Gamma(1+\alpha \xi)}, \quad \xi \in[0,1] .
$$

The right-hand side of the above inequality tends to zero as $\lambda, \delta \rightarrow 0$. So we can deduce that $\left\|\left(\Psi_{2} x\right)(t)-\left(\Psi_{2}^{\lambda, \delta} x\right)(t)\right\|_{C_{\alpha}} \rightarrow 0$ as $\lambda, \delta \rightarrow 0^{+}$which enables us to claim that there are relatively compact sets arbitrarily close to the set $V(t)=\left\{\left(\Psi_{2} x\right)(t), x \in B_{r}\right\}$. Hence, $V(t)=$ $\left\{\left(\Psi_{2} x\right)(t), x \in B_{r}\right\}$ is relatively compact in $X$.

We deduce, from Claims 1-3 and the Arzola-Ascoli theorem, that $\Psi_{2}$ is a completely continuous map. Using Krasnoselskii's fixed point theorem, we claim that the operator equation $\Psi x=\Psi_{1} x+\Psi_{2} x$ has a fixed point on $B_{r}$ which is a mild solution for system (1.1). The proof is complete.

To give our last existence theorem, we require the following hypotheses:

$\left(\mathrm{H}_{0}\right) S(t)$ is continuous in the uniform operator topology for $t \geq 0$, and $\{S(t)\}_{t \geq 0}$ is uniformly bounded, i.e., there exists $M \geq 1$ such that $\sup _{t \in[0,+\infty)}|S(t)| \leq M$;

$\left(\mathrm{H}_{6}\right)$ there exists positive constant $L$ such that for any $x_{1}, x_{2} \in C_{\alpha}(J$, $X)$, we have $E \| g\left(x_{1}\right)$ $g\left(x_{2}\right)\left\|^{2} \leq L\right\| x_{1}-x_{2} \|_{C_{\alpha}} ;$

$\left(\mathrm{H}_{7}\right)$ there exists a function $N_{1}(t) \in L^{\frac{1}{2 \alpha_{1}-1}}(J), \alpha_{1} \in\left[\frac{1}{2}, \alpha\right)$, such that for any $x, y \in X, t \in J$, we have

$$
E\|F(t, x(t))-F(t, y(t))\|^{2} \leq N_{1}(t) \cdot\|x-y\|_{C_{\alpha}} .
$$

For convenience, let

$$
\begin{aligned}
M^{\prime}= & 4 b^{2(1-\alpha)} \frac{M^{2}}{\Gamma^{2}(\alpha)} L+4 b^{2(1-\alpha)} M_{0}^{2} L+4 b^{2(1-\alpha+\alpha \beta)} K^{2}(\alpha, \beta) L \\
& +4 b^{2(1-\alpha)} \operatorname{Tr} Q \frac{M^{2}}{\Gamma^{2}(\alpha)} \Lambda\left\|N_{1}\right\|_{L^{2 \alpha_{1}-1}} .
\end{aligned}
$$

To end this section, we shall proceed with our last existence and uniqueness theorem for system (1.1) based on the Banach contraction principle.

Theorem 3.2 Assume that hypotheses $\left(\mathrm{H}_{0}\right),\left(\mathrm{H}_{2}\right)-\left(\mathrm{H}_{7}\right)$ hold, then system (1.1) has a unique mild solution on $B_{r}$ provided that $M^{\prime}<1$.

Proof Define operator $\Psi$ as in Theorem 3.1. Then by similar arguments employed in Theorem 3.1, we can get that operator $\Psi$ maps $B_{r}$ into itself, where $B_{r}$ is defined as in Theorem 3.1.

Moreover, we have

$$
\begin{aligned}
& \|(\Psi x)(t)-(\Psi y)(t)\|_{C_{\alpha}}^{2} \\
& =\sup _{t \in J^{\prime}} t^{2(1-\alpha)} E\|(\Psi x)(t)-(\Psi y)(t)\|^{2} \\
& \leq 4 \sup _{t \in J^{\prime}} t^{2(1-\alpha)} E\left\|\mathcal{P}_{\alpha}(t)[g(x)-g(y)]\right\|^{2}
\end{aligned}
$$




$$
\begin{aligned}
& +4 \sup _{t \in J^{\prime}} t^{2(1-\alpha)} E\|h(t, x(t))-h(t, y(t))\|^{2} \\
& +4 \sup _{t \in J^{\prime}} t^{2(1-\alpha)} E\left\|\int_{0}^{t}(t-s)^{\alpha-1} A \mathcal{P}_{\alpha}(t-s)[h(s, x(s))-h(s, y(s))] d s\right\|^{2} \\
& +4 \sup _{t \in J^{\prime}} t^{2(1-\alpha)} E\left\|\int_{0}^{t}(t-s)^{\alpha-1} \mathcal{P}_{\alpha}(t-s)[F(s, x(s))-F(s, y(s))] d \omega(s)\right\|^{2} \\
& \leq 4 \sup _{t \in(0, b]} t^{2(1-\alpha)} \frac{M^{2}}{\Gamma^{2}(\alpha)} E\|g(x)-g(y)\|^{2} \\
& +4 \sup _{t \in J^{\prime}} t^{2(1-\alpha)}\left\|A^{-\beta}\right\|^{2} E\left\|A^{\beta} h(t, x(t))-A^{\beta} h(t, y(t))\right\|^{2} \\
& +4 \sup _{t \in J^{\prime}} t^{2(1-\alpha)} \int_{0}^{t}\left\|(t-s)^{\alpha-1} A^{1-\beta} P_{\alpha}(t-s)\right\| d s \\
& \times \int_{0}^{t}(t-s)^{\alpha-1} A^{1-\beta} \mathcal{P}_{\alpha}(t-s) E\left\|A^{\beta} h(s, x(s))-A^{\beta} h(s, y(s))\right\|^{2} d s \\
& +4 \sup _{t \in(0, b]} \operatorname{Tr} Q \frac{M^{2}}{\Gamma^{2}(\alpha)} \int_{0}^{t}(t-s)^{2 \alpha-2} E\|F(s, x(s))-F(s, y(s))\|^{2} d s \\
& \leq 4 b^{2(1-\alpha)} \frac{M^{2}}{\Gamma^{2}(\alpha)} L\|x-y\|_{C_{\alpha}}+4 b^{2(1-\alpha)} M_{0}^{2} L\|x-y\|_{C_{\alpha}} \\
& +4 b^{2(1-\alpha+\alpha \beta)} K^{2}(\alpha, \beta) L\|x-y\|_{C_{\alpha}} \\
& +4 b^{2(1-\alpha)} \operatorname{Tr} Q \frac{M^{2}}{\Gamma^{2}(\alpha)} \frac{b^{(1+c)\left(2-2 \alpha_{1}\right)}}{(1+c)^{2-2 \alpha_{1}}}\left\|N_{1}\right\|_{L^{\frac{1}{2 \alpha_{1}-1}}}\|x-y\|_{C_{\alpha}} \\
& =\left[4 b^{2(1-\alpha)} \frac{M^{2}}{\Gamma^{2}(\alpha)} L+4 b^{2(1-\alpha)} M_{0}^{2} L+4 b^{2(1-\alpha+\alpha \beta)} K^{2}(\alpha, \beta) L\right. \\
& \left.+4 b^{2(1-\alpha)} \operatorname{Tr} Q \frac{M^{2}}{\Gamma^{2}(\alpha)} \Lambda\left\|N_{1}\right\|_{L^{\frac{1}{2 \alpha_{1}-1}}}\right]\|x-y\|_{C_{\alpha}} \\
& <\|x-y\|_{C_{\alpha}} .
\end{aligned}
$$

Hence, the Banach contraction principle implies that $\Psi$ has a unique fixed point in $B_{r}$ which is a mild solution for system (1.1). This completes the proof.

\section{An example}

As an application, we present an example to illustrate our results. Consider the following fractional stochastic evolution equation driven by both $\mathrm{fBm}$ and Wiener process:

$$
\left\{\begin{aligned}
&{ }^{L} \mathcal{D}^{\frac{3}{4}}\left[u(t, z)-\int_{0}^{\pi} W(z, y) u(t, y) d y\right]= u_{z z}(t, z)+\hat{F}\left(t, u(t)(z) \frac{d \omega(t)}{d t},\right. \\
&+\hat{\sigma}\left(t, u(t)(z) \frac{d B_{Q}^{H}(t)}{d t}, \quad t \in(0, b], z \in[0, \pi],\right. \\
& u(t, 0)=u(t, \pi)=0, \quad t \in J=[0, b], \\
& \mathcal{I}^{\frac{1}{4}}\left(u(t, z)-\sum_{i=1}^{n} \int_{0}^{\pi} k(z, y) u\left(t_{i}, y\right) d y\right)=u_{0}(z), \quad z \in[0, \pi],
\end{aligned}\right.
$$

where ${ }^{L} \mathcal{D}^{\frac{3}{4}}$ denotes the Riemann-Liouville fractional derivative of order $\frac{3}{4}, \mathcal{I}^{\frac{1}{4}}$ is a Riemann-Liouville integral of order $\frac{1}{4}, n$ is a positive integer, $0<t_{0}<t_{1}<\cdots<t_{n}<b$, $z \in[0, \pi]$. 
To write the above system (4.1) into the abstract form of (1.1), we choose the space $X=L^{2}[0, \pi]$. Next we define an operator $A$ by $A v=v^{\prime \prime}$ with the domain $D(A)=\{v \in X$ : $v, v^{\prime}$ absolutely continuous, $\left.v^{\prime \prime} \in X, v(0)=v(\pi)=0\right\}$. Then $A$ is a generator of a strongly continuous semigroup $\left\{S(t)_{t \geq 0}\right\}$ which, as we know, is compact, analytic, and self-adjoint.

Moveover, from the previous work [25], $A$ has a discrete spectrum, the eigenvalues of $A$ are $-n^{2}, n \in N$ with corresponding orthogonal eigenvectors $e_{n}(z)=\sqrt{\frac{2}{\pi}} \sin (n z)$. Then $A z=\sum_{n=1}^{\infty} n^{2}\left\langle z, e_{n}\right\rangle e_{n}$. On the other hand, we know that

(i) for each $v \in X, S(t) v=\sum_{n=1}^{\infty} e^{-n^{2} t}\left\langle v, e_{n}\right\rangle e_{n}$, in addition, $S(\cdot)$ is a uniformly stable semigroup and $\|S(t)\| \leq e^{-t}$;

(ii) for each $v \in X, A^{-\frac{1}{2}} v=\sum_{n=1}^{\infty} \frac{1}{n}\left\langle v, e_{n}\right\rangle e_{n}$ and $\left\|A^{-\frac{1}{2}}\right\|^{2}=1$;

(iii) the operator $A^{\frac{1}{2}}$ is given by $A^{\frac{1}{2}} v=\sum_{n=1}^{\infty} n\left\langle v, e_{n}\right\rangle e_{n} \in X$ on the space $D\left(A^{\frac{1}{2}}\right)=\left\{v(\cdot) \in X, \sum_{n=1}^{\infty}\left\langle v, e_{n}\right\rangle e_{n} \in X\right\}$.

Letting $\left(W_{h} v\right)(z)=\int_{0}^{\pi} W(z, y) v(y) d y$, for $v \in E=L^{2}([0, \pi], \mathbb{R}), z \in[0, \pi]$, we assume that the following assumptions hold:

$(\bar{a}) \omega(s)$ denotes a one-dimensional standard Brownian motion;

$(\bar{b})$ the function $W(z, y), z, y \in[0, \pi]$ is measurable and

$$
\int_{0}^{\pi} \int_{0}^{\pi} W^{2}(z, y) d y d z<\infty
$$

$(\bar{c})$ the function $\partial_{z} W(z, y)$ is measurable, $W(0, y)=W(\pi, y)=0$, and

$$
\bar{\Lambda}=\left(\int_{0}^{\pi} \int_{0}^{\pi}\left(\partial_{z} W(z, y)\right)^{2} d y d z\right)^{\frac{1}{2}}<L<\infty .
$$

Note that $(\bar{c})$ enables us to show that $W_{h}$ is a bounded linear operator on $X$. Furthermore, $W_{h}(v) \in D\left(A^{\frac{1}{2}}\right)$, and $\left\|A^{\frac{1}{2}} W_{h}\right\|_{L^{2}[0, \pi]}<\infty$. Indeed, the definition of $W_{h}$ and (ii) yield that

$$
\left\langle W_{h}(v), e_{n}\right\rangle=\int_{0}^{\pi} e_{n}(z)\left(\int_{0}^{\pi} W(z, y) v(y) d y\right) d z=\frac{1}{n} \sqrt{\frac{2}{\pi}}\langle\bar{W}(v), \cos (n z)\rangle,
$$

where $\bar{W}$ is defined by

$$
(\bar{W}(v))(z)=\int_{0}^{\pi} W_{z}(z, y) v(y) d y
$$

We know from $(\bar{b})$ that $\bar{W}: X \rightarrow X$ is a bounded linear operator with $\|\bar{W}\|_{L^{2}[0 . b]} \leq \bar{\Lambda}$. Hence we can write $\left\|A^{\frac{1}{2}} W_{h}(v)\right\|_{L^{2}[0, \pi]}^{2}=\|\bar{W}(v)\|_{L^{2}[0, \pi]}^{2}$.

(iv) For the function $\hat{F}:[0, b] \times[0, \pi] \rightarrow[0, \pi]$ the following conditions are satisfied:

(1) for each $s \in[0, b], \hat{F}(s, \cdot)$ is continuous, and for each $u \in X, \hat{F}(\cdot, u)$ is measurable;

(2) there exist a function $N(t) \in L^{\frac{1}{2 \alpha_{1}-1}}(J), \alpha_{1} \in\left[\frac{1}{2}, \alpha\right)$ and a continuous

nondecreasing function $\vartheta:[0, \infty) \rightarrow(0, \infty)$ such that for any $(t, u) \in J \times X$, we have $E\|F(t, u(t))\|^{2} \leq N(t) \times \vartheta\left(\|u\|_{C_{1-\alpha}}\right), \liminf _{r \rightarrow \infty} \frac{\vartheta(r)}{r} d s=\Theta<\infty$.

Define the fractional Brownian motion in $Y$ by

$$
\left.B_{Q}^{H}(t)=\sum_{n=1}^{\infty} \sqrt{(} \lambda_{n}\right) B_{n}^{H}(t) e_{n}
$$


where $H \in\left(\frac{1}{2}, 1\right)$ and $\left\{B_{n}^{H}, n \in N\right\}$ is a sequence of one-dimensional fractional Brownian motions which are mutually independent. Let us assume that the function $\hat{\sigma}:[0,+\infty) \rightarrow$ $\mathcal{L}_{2}^{0}\left(L^{2}([0,1]), L^{2}([0,1])\right)$ satisfies

$$
\int_{0}^{b}\|\hat{\sigma}(s)\|_{L_{0}^{2}}^{\frac{2}{2 \alpha_{1}-1}} d s<\infty, \quad \forall b>0 .
$$

Letting $u(t)(z)=u(t, z), t \in J, z \in[0, \pi]$, we define $h:[0, b] \times X \rightarrow X, F:[0, b] \times X \rightarrow$ $L(K, X)$ and $g: E \rightarrow X$ by $h(t, u)=W_{h}(u), \hat{F}(s, u)(z)=F(s, u(z)), g(u)=\sum_{i=0}^{p} \bar{Q} u\left(t_{i}\right)$ and $\sigma(t)=\hat{\sigma}(t)$, respectively, where

$$
\bar{Q}(u)=\int_{0}^{\pi} k(y, z) u(y) d y .
$$

With the above choices of $A, F, h, \sigma$, system (4.1) can be seen as an abstract form of system (1.1). On the other hand, suppose that the assumptions of Theorem 3.1 hold. Thus, using Theorem 3.1, we claim that system (4.1) admits a mild solution on $[0, b]$ under the above additional assumptions.

\section{Conclusion}

In this paper, we have considered a class of Riemann-Liouville fractional stochastic evolution equations. In particular, the fixed point technique, fractional calculus, and stochastic analysis were used for achieving the sufficient conditions to ensure the existence of mild solutions of Riemann-Liouville fractional stochastic evolution equations driven by Wiener process and $\mathrm{fBm}$. We last gave an illustrative example. Our future work will address the explosive solutions of fractional Navier-Stokes stochastic differential equations driven by $\mathrm{fBm}$ and multiplicative noise.

\section{Funding}

This work is supported by National Natural Science Foundation of China (Grant Nos. 12001393, 11961069), Natural Science Foundation of Shanxi (201901D211103), Natural Science Foundation of Xinjiang (2019D01A71), Scientific Research Programs of Colleges in Xinjiang (XJEDU2018Y033), Outstanding Young Science and technology personnel Training program of Xinjiang(2019Q022).

Availability of data and materials

Not applicable.

Competing interests

The authors declare that they have no competing interests.

Authors' contributions

All authors have contributed equally. All authors read and approved the final manuscript.

\section{Author details}

${ }^{1}$ College of Mathematics, Taiyuan University of Technology, 030024 Taiyuan, China. ${ }^{2}$ School of Mathematics Sciences, Xinjiang Normal University, 830017 Urumqi, China.

\section{Publisher's Note}

Springer Nature remains neutral with regard to jurisdictional claims in published maps and institutional affiliations.

Received: 22 July 2020 Accepted: 28 December 2020 Published online: 11 January 2021

\section{References}

1. Ahmed, H.M., El-Borai, M.M.: Hilfer fractional stochastic integro-differential equations. Appl. Math. Comput. 331(1), 182-189 (2018)

2. Byszewski, L., Lakshmikantham, V.: Theorem about the existence and uniqueness of a solution of a nonlocal abstract Cauchy problem in a Banach space. Appl. Anal. 40(1), 11-19 (1991) 
3. Cui, J., Yan, L.: Existence result for fractional neutral stochastic integro-differential equations with infinite delay. J. Phys. A, Math. Theor. 44(33), 335201 (2011)

4. Deng, S.F., Shu, X.B., Mao, J.Z.: Existence and exponential stability for impulsive neutral stochastic functional differential equations driven by fBm with noncompact semigroup via Monch fixed point. J. Math. Anal. Appl. 467(1), 398-420 (2018)

5. Furati, K.M., Kassim, M.D., Tatar, N.e-.. Existence and uniqueness for a problem involving Hilfer factional derivative. Comput. Math. Appl. 64(6), 1612-1626 (2012)

6. Gu, H.B., Trujillo, J.J.: Existence of mild solution for evolution equation with Hilfer fractional derivative. Appl. Math. Comput. 257(1), 344-354 (2015)

7. Hilfer, R.: Applications of Fractional Calculus in Physics. World Scientific, Singapore (2000)

8. Kilbas, A.A., Srivastava, H.M., Trujillo, J.J.: Theory and Application of Fractional Differential Equations. North-Holland Mathematics Studies, vol. 204. Elsevier, Amsterdam (2006)

9. Lakhel, E., McKibben, M.A.: Existence of solutions for fractional neutral functional differential equations driven by fBm with infinite delay. Stochastics 90(3), 313-329 (2018)

10. Li, K.X., Peng, J.G., Jia, J.X.: Cauchy problems for fractional differential equations with Riemann-Liouville fractional derivatives. J. Funct. Anal. 263(2), 476-510 (2012)

11. Lin, A., Hu, L.: Existence results for impulsive neutral stochastic functional integro-differential inclusions with nonlocal initial conditions. Comput. Math. Appl. 59(1), 64-73 (2010)

12. Liu, Y.L., Lv, J.Y.: Existence results for Riemann-Liouville fractional neutral evolution equations. Adv. Differ. Equ. 2014(1), 83 (2014)

13. Liu, Z.H., Li, X.W.: Existence and uniqueness of solutions for the nonlinear impulsive fractional differential equations. Commun. Nonlinear Sci. Numer. Simul. 18(6), 1362-1373 (2013)

14. Mainardi, F.: Fractional Calculus: Some Basic Problems in Continuum and Statistical Mechanics. CISM Courses and Lectures, vol. 378. Springer, Vienna (1997)

15. Miller, K.S., Ross, B.: An Introduction to the Fractional Calculus and Differential Equations. Wiley, New York (1993)

16. Mophou, G.M.: Existence and uniqueness of mild solutions to impulsive fractional differential equations. Nonlinear Anal. TMA 72(3), 1604-1615 (2010)

17. Podlubny, l.: Fractional Differential Equations. Academic Press, San Diego (1999)

18. Sakthivel, R., Revathi, P., Ren, Y.: Existence of solutions for nonlinear fractional stochastic differential equations. Nonlinear Anal. TMA 81(1), 70-86 (2013)

19. Shu, X.B., Lai, Y., Chen, Y.: The existence of mild solutions for impulsive fractional partial differential equations, Nonlinear Anal. TMA 74(5), 2003-2011 (2011)

20. Wang, R.N., Chen, D.H., Xiao, T.J: Abstract fractional Cauchy problems with almost sectorial operators. J. Differ. Equ. 252(1), 202-235 (2012)

21. Xu, L.P., Li, Z.: Stochastic fractional evolution equations with fractional Brownian motion and infinite delay. Appl. Math. Comput. 336(1), 36-46 (2018)

22. Yang, M., Wang, Q.R.: Approximate controllability of Riemann-Liouville fractional differential inclusions. Appl. Math. Comput. 274(1), 267-281 (2016)

23. Zhou, Y.: Basic Theory of Fractional Differential Equations. World Scientific, Singapore (2014)

24. Zhou, Y: Attractivity for fractional evolution equations with almost sectorial operators. Fract. Calc. Appl. Anal. 21(3), 786-800 (2018)

25. Zhou, Y., Jiao, F.: Existence of mild solutions for fractional neutral evolution equations. Comput. Math. Appl. 59(3), 1063-1077 (2010)

26. Zhou, Y., Zhang, L., Shen, X.H.: Existence of mild solutions for fractional evolution equations. J. Integral Equ. Appl. 25(4), 557-585 (2013)

27. Zou, G.A.: A Galerkin finite element method for time-fractional stochastic heat equation. Comput. Math. Appl. 75(11), 4135-4150 (2018)

28. Zou, G.A., Wang, B.: Solitary wave solutions for nonlinear fractional Schrödinger equation in Gaussian nonlocal media. Appl. Math. Lett. 88(1), 50-57 (2019)

\section{Submit your manuscript to a SpringerOpen ${ }^{\circ}$ journal and benefit from:}

- Convenient online submission

- Rigorous peer review

- Open access: articles freely available online

- High visibility within the field

Retaining the copyright to your article

Submit your next manuscript at $\boldsymbol{~ s p r i n g e r o p e n . c o m ~}$ 